De la crise de la diaconie à la diaconie de la crise : quelques pistes pour l'avenir

\section{Pierre de Salis}

Ma contribution va s'articuler en deux temps, conformément à son titre. Dans la première partie, concernant «la crise de la diaconie», je vais évoquer, à partir des recherches récentes, ${ }^{1}$ les enjeux liés à la diaconie dans les Églises cantonale de Suisse, enjeux marqués par un certain nombre de contradictions et de paradoxes. Dans la deuxième partie, concernant « la diaconie de crise : quelques pistes pour l'avenir», je formulerai quelques propositions d'action.

Le point de vue qui est le mien est double. Il y a d'une part celui du formateur chargé de la formation professionnelle et théologique des futurs diacres au sein des Églises de Suisse romande et d'autre part celui d'un chercheur engagé dans un projet doctoral en théologie et en histoire des origines du christianisme, plus précisément sur les lettres de Paul et la pragmatique de la communication.

A l'heure actuelle, revient régulièrement le refrain que l'Église doit se réinventer. Au gré des analyses sociologiques, des sondages d'opinion, des blogs ou des micros-trottoirs, la conclusion qui ressort est que pour survivre, les Églises doivent s'adapter à la société qui change. Comment ne pas être d'accord? La vie cultuelle, activité centrale de l'Église, est en sérieuse perte de vitesse et il faut faire quelque chose, mais quoi ? C'est bien connu: ces transformations nous renvoient globalement aux profondes mutations sociales des années 1960, avec l'augmentation du niveau de vie, l'affirmation des libertés individuelles, etc. L'émergence des

1 Voir Jörg Stolz/Edmée Ballif, L’avenir des Réformés. Les Églises face aux changements sociaux, Genève 2011, en particulier 101-111 et 153-157.

Jahrbuch Diakonie Schweiz 1 (2017) - ISSN 2504-3994

Dieser Text ist lizenziert unter einer Creative Commons Namensnennung 4.0 International Lizenz (CC BY 4.0): (https://creativecommons.org/licenses/by/4.0/). nouvelles technologies de l'information et de la communication (années 1990 et 2000) a intensifié massivement cette tendance, laquelle génère une versatilité et une mobilité tout azimut (liberté de croyance et recentrement sur l'individu, ère du zapping, ère du syncrétisme ${ }^{2}$ religieux ou de ce qu'on désigne parfois comme le buffet religieux). L'individu devient instance dernière de vérité, en particulier en matière de convictions religieuses. On a l'impression aujourd'hui que parler de développement spirituel est devenue la manière politiquement correcte de promouvoir la religion. Parler de confort spirituel personnel, c'est beaucoup plus agréable - et surtout plus facile - que de chercher à recruter des candidats/es au catéchisme ou des lèves-tôt pour aller au culte le dimanche à 10 heures !

En 2011 a eu lieu à Montmirail un important forum pour faire le point sur la situation de la diaconie en Suisse. ${ }^{3}$ Le constat fut clair : la société va de plus en plus mal et l'engagement diaconal de l'Église, engagement par essence social et prophétique, est plus que jamais nécessaire. Mais celui-ci doit être repensé, modernisé et mieux soutenu, tant institutionnellement que spirituellement. Ce forum avait pour but de dresser l'état général de la diaconie et d'esquisser des pistes concrètes d'action. Ce qui fut fait sous la forme des Dix propositions aux Eglises (10 Empfehlungen an die Kantonalkirchen). Couplées aux conclusions du Forum de Montmirail, les analyses récentes relatives à la situation des Églises en Suisse montrent que la diaconie est aujourd'hui aux prises avec quatre grands défis majeurs, ${ }^{4}$ lesquels signalent autant de symptômes de la crise de la diaconie :

2 Le terme de syncrétisme est assurément galvaudé et son usage sémantique dans l'opinion publique guère régulé !

3 Voir le cahier (intitulé Diakonia) consacré au Forum de Montmirail de 2011, dans Inter Pares, Bulletin de la société pastorale suisse 3 (2011), 3-21 (les dix propositions aux Églises se trouvent aux pages 20f.)

4 Voir Stolz/Ballif (cf. note 1), 153-157. 
Premier défi : le problème confessionnel. Si l'opinion publique salue volontiers que l'action sociale des Églises remplit une mission d'intérêt général, un accent confessionnel trop marqué peut péjorer son action. L'aide sociale est devenue une action très complexe et elle requiert des compétences professionnelles, notamment en sciences sociales, en psychologie et en droit. Toutefois le personnel d'Église n'est pas formé pour cela. Nous le voyons dans la formation diaconale que nous dispensons en Suisse romande. Les diacres sont formés au travail en réseau et à la collaboration, comme spécialistes du lien social. L'objectif de la formation est de leur permettre d'acquérir une solide connaissance des réseaux et des ressources de l'action diaconale. Leur mission devient davantage une responsabilité d'orientation. Dit autrement, sentir le point limite à partir duquel il convient de passer la main aux services sociaux, aux services d'aide psychologique ou aux services juridiques (on notera en passant la récurrence de la notion de service).

Deuxième défi : le problème professionnel. S'il est une chose en Suisse romande qui a porté ses fruits après le Forum 2011 de Montmirail, c'est bien celui de la formation des diacres, lesquels disposent d'un dispositif de formation reposant sur un profil professionnel clair et visant à l'acquisition de compétences professionnelles polyvalentes avec d'autres métiers orientés sur les relations humaines : écoute et accompagnement de personnes individuelles ou de groupes, travail par objectifs, gestion de projets, gestion de conflits, animation communautaire, travail en réseau, etc. Les ministères diaconaux, dès leur mise en place en Suisse romande il y a une cinquantaine d'années, ont connu une trajectoire chaotique: l'enthousiasme prophétique des débuts s'est essoufflé, et ceci en raison principalement d'aspirations contradictoires : d'une part, les diacres ont représenté pour les Églises une main d'œuvre professionnelle bienvenue face à la pénurie pastorale et cela n'a pas été simple pour les diacres véritablement au bénéfice d'une vocation au travail social dans l'Église de s'affirmer en tant que tels. D'autre part, pour de nombreuses personnes, la création d'un ministère diaconal a représenté une porte ouverte pour le travail professionnel dans l'Eglise aux personnes qui n'avaient pas accès à l'université, mais qui étaient habitées par une vocation pastorale. En Suisse allemande, on rencontre des tensions analogues, entre une conception privilégiant le travail social laïque et la consécration au ministère diaconal. Certaines Églises consacrent les diacres (Argovie, Grisons, Saint-Gall, Schaffhouse, Soleure, Thurgovie), d'autres pas (les deux Appenzell, Bâle-Campagne, Nidwald, Obwald, Tessin, Uri, Zoug, Zurich). D'autres enfin prévoient les deux possibilités (Berne-Jura-Soleure, BâleVille, Glaris, Lucerne, Schwyz).

Troisième défi : le problème économique. Les Églises cantonales de Suisse sont toutes ou presque confrontées à la nécessité de faire des économies (les raisons sont multiples : baisse des rentrées financières, de par la baisse du nombre de personnes se déclarant protestantes - et en particulier du nombre de croyants pratiquant -, baisses sévères de revenus en particulier pour les Églises cantonales séparés de l'État, réformes fiscales, votations cantonales visant la séparation de l'Église et de l'État, etc.). La tendance générale est à la baisse un peu partout. Les Églises, comme les cantons, se trouvent contraintes de réduire la voilure aussi parmi les activités à caractère social ou solidaire.

Quatrième défi : le problème de la relève professionnelle. Il n'est pas évident, pour les jeunes aujourd'hui, de choisir d'embrasser une carrière professionnelle dans l'Église. La perte de vitesse institutionnelle, les campagnes financières, les diminutions fracassantes d'effectifs, une gestion des ressources humaine parfois stressante, le peu de garantie de perspectives d'emploi stable à long terme, tout cela ne favorise pas franchement l'émergence de nouvelles vocations. Ces enjeux sont à situer dans le contexte général de la transformation du croire, en particulier parmi les jeunes générations.

Esquisser des pistes pour l'avenir de la diaconie est un exercice délicat, car il convient de ne pas sombrer dans la banalité ni de prêcher des généralités qu'au fond tout le monde connaît. Ma proposition - à l'heure du feu d'artifice tout azimut des festivités du $500^{\text {e }}$ anniversaire de la Réforme du XVIe siècle - est de retourner aux sources. Ceci en particulier par 
un retour au cour du patrimoine cher à notre conscience protestante, le principe du sola scriptura!

Il est toujours stimulant de relire les textes sources et de confronter nos idées, nos conclusions ou nos évidences avec les racines historiques du sens des mots. Autant le concept de diaconie paraît relever de l'évidence (le service du prochain, l'attention et l'action en faveur des personnes en situation de fragilité), autant le retour aux origines du mot révèle quelques surprises, lesquelles sont autant de stimulations pour évaluer la diaconie aujourd'hui.

Quel est le sens étymologique premier du mot grec diakonia? Celui-ci résulte de l'assemblage d'une préposition (dia qui signifie « à travers ») et d'un mot (konia qui signifie «la poussière »). ${ }^{5}$ Le verbe qui est lié à ce substantif signifie « se rouler dans la poussière ", pour parler, par exemple, d'un cheval qui se roule dans la poussière (parce que cela gratte ou pour éliminer les parasites présents dans son pelage ?) ou d'un athlète qui se frotte de poussière avant la compétition (on peut penser ici à la récente fête fédérale de lutte à Estavayer-le-Lac !). Ce sens premier, au delà du sens usuel de service et de serviteur préfigure tout un programme théologique. ${ }^{6}$ Il souligne le caractère terre-à-terre de la diaconie. Celle-ci prend le sacré à rebrousse-poil, si on ose dire les choses ainsi! Le sacré s'emploie à codifier le bien et le mal, à réglementer le pur et l'impur et à fixer les principes du culte et les règles de vie de la communauté et à réguler la compréhension des croyances. La diaconie renvoie à la poussière, à la

5 Voir Fritz Lienhard, "Diaconie», dans Bernard Kaempf (éd.), Introduction à la théologie pratique, Strasbourg 1997, 259-274 (pour les considérations étymologiques, voir en particulier 259-261).

6 «Ainsi le choix de ce terme dans le Nouveau Testament pour désigner le service d'autrui donne à celui-ci un caractère prosaïque qui le distingue de la lâtrie et de la liturgie et lui confère une dimension polémique vis-à-vis du sacré qui distingue le pur et l'impur, excluant le mal, là où la diaconie le prend en charge pour le surmonter » selon Fritz Lienhard (cf. note 3), 259f. (c'est l'auteur qui souligne). chute à terre, à la lutte, à l'horizontalité. Se rouler dans la poussière fait écho au combat de Jacob avec l'ange (Genèse 32,25: « un homme se roula avec lui dans la poussière jusqu'au lever de l'aurore »), un combat qui porte indéfectiblement la marque, dans la chair de Jacob, de la dimension dramatique de la condition humaine. Mais la poussière renvoie également aux paroles sévères de Jésus envoyant ses disciples en mission, en leur recommandant de secouer la poussière de leurs sandales quand ils quittent un lieu qui leur a réservé un mauvais accueil (Matthieu 10,11-14: "dans quelque ville ou village que vous entriez, informez-vous pour savoir qui est digne de vous recevoir et demeurez là jusqu'à votre départ. En entrant dans la maison, saluez-la; si cette maison en est digne, que votre paix vienne sur elle; mais si elle n'en est pas digne, que votre paix revienne à vous. Si l'on ne vous accueille pas et si l'on écoute pas vos paroles, en quittant cette maison ou cette ville, secouez la poussière de vos pieds »).

Dans la culture grecque, le terme diaconia recèle une connotation pas très positive, désignant grosso modo le service domestique de base. Le Nouveau Testament va opérer un virage spectaculaire, en réorientant christologiquement cette notion de serviteur à la lumière de la posture du serviteur souffrant pour sauver les innocents de la souffrance (cf. Ésaïe 52,12-53,12 : le poème du serviteur souffrant). Cette réorientation, bien connue met en lien la diaconie et la théologie de la croix : le serviteur souffrant et obéissant, obéissant jusqu'à la mort sur la croix.

Si on passe brièvement en revue les quatre symptômes de la crise et qu'on cherche à esquisser des pistes d'action diaconales pour l'avenir des pistes «à travers la poussière »-des pistes à la croisée des chemins entre bénédiction et souffrance, qu'est-ce que cela peut donner?

Pour le premier défi, celui des enjeux confessionnels, je dirais qu'il y a un important travail de sensibilisation à faire, valorisant le caractère non prosélyte d'une action sociale d'Église. Dit autrement, toute l'action sociale en paroisse, dans la cité, dans la rue, à l'école, à l'hôpital, en prison, etc. est un service d'intérêt public, contribuant, au nom des valeurs chrétiennes ouvertement référencées comme telles, au service de son 
prochain, fut-il originaire de Samarie. Un service ouvert et privilégiant le travail en réseau avec toutes les forces vives de la société civile engagées dans l'action sociale et la solidarité active.

Pour le deuxième défi, celui des enjeux professionnels, je dirais qu'il y a un important travail de renforcement des identités et des compétences respectives des forces vives engagées dans le travail ecclésial, pasteurs, diacres, travailleurs sociaux, et autres professionnels (direction, médias, formation, ressources humaines, etc.). La diaconie doit jouer à fond d'une part la carte de la remise en question, entre repérage et analyse des besoins actuels (ainsi notre journée consacrée à Foi et protection des réfugiés) et d'autre part celle de la vocation de porter au cœur de la vie cultuelle et communautaire le souci pour les personnes en situation de fragilité.

Pour le troisième, celui des enjeux économiques, je dirais que la diaconie doit continuer à faire entendre la voix des sans-voix dans nos pratiques et conduites d'Églises. Une Église qui renoncerait à la diaconie, que lui reste-t-elle? Il convient aussi de favoriser les collaborations et les échanges d'expérience. Faire des choix et chercher à mutualiser les principaux projets diaconaux avec les Églises sœurs, voire même avec l'action sociale prônée par les croyants d'autres religions.

Pour le quatrième défi, celui de la relève professionnelle, je dirais que la diaconie doit également s'occuper des questions et des défis intergénérationnels. A l'heure actuelle, les habitudes de vie des jeunes générations se sont radicalement transformées par rapport à celles qui les ont précédé. Les taux élevés de suicide chez les jeunes, les difficiles perspectives d'emploi, un monde pollué comme jamais, le tableau est sombre, mais «se rouler dans la poussière » passe par la prise en compte, dans l'action diaconale, du caractère crucial de ces inquiétantes perspectives. Le défi est immense mais l'action diaconale témoigne de réussites fort encourageantes: groupes de partage pour familles monoparentales, projets favorisant les liens multiculturels, lieux de rencontres pour personnes

isolées, autant d'initiatives «dans la poussière du monde» exprimant la solidarité et fabriquant des liens renouvelés entre les individus et la communauté.

Pour terminer, je dirais que je souscris volontiers à cette parole attribuée à Ignace de Loyola, que je trouve emblématique pour conclure nos propos : "prie comme si tout dépendait de Dieu, et agis comme si tout dépendait de toi $\gg .^{7}$ Le redire dans une perspective diaconale et chrétienne revient à le formuler en «nous»: "prions comme si tout dépendait de Dieu, agissons comme si tout dépendait de nous ».

Auteur:

Pierre de Salis,

pasteur, responsable de la formation diaconale de l'Office protestant de formation (opf) Loyola 1998 (cité par Benoit XVI) : « agis comme si tout dépendait de toi, en sachan qu'en réalité tout dépend de Dieu » (Angelus 17 juin 2012). Je remercie Steeve Bélanger pour cette précision 